\title{
REMOTE SENSING OF ATMOSPHERIC TURBULENCE PROFILES BY LASER GUIDE STARS Xiwen Qiang
}

\author{
The State Key Laboratory of Laser Interaction with Matter, Xi'an 710024, P. R. China
}

\begin{abstract}
Ranged-resolved profiles of atmospheric turbulence are necessary and important for many applications in astronomical and adaptive optics communities. In order to characterize the vertical atmospheric structure in field, a technique is put forward to remote sensing ranged-resolved profiles of atmospheric turbulence by combined with laser guide stars and differential image motion method. Laser guide stars are formed at several successive altitudes by projecting pulsed laser, returned signals of images are received by a optical system with two receiving telescopes, and variance of centroids' distance is derived from the images with two spots at the same altitude. So, based on a inversion algorithm, atmospheric turbulence profiles are retrieved from differential image motion variance of distance of centroids at various altitudes. The structure constants of refractive index of atmosphere range from $10^{-14} \mathrm{~m}^{-2 / 3}$ at lower altitudes to $10^{-16} \mathrm{~m}^{-2 / 3}$ at higher altitudes are remote sensed experimentally. The results show it is a effective method that combined laser guide stars with differential image motion method and could sense atmospheric turbulence profiles remotely in real time.
\end{abstract}

KEYWORDS atmospheric turbulence profiles, remote sensing, atmospheric optics, laser guide stars, differential image motion method

\section{INTRODUCTION}

Characteristics of atmospheric turbulence varies temporally and spatially, so remote sensing of atmospheric turbulence profiles in real time is important and necessary for applications such as performance analysis of astronomical adaptive optics systems, astronomical sites surveys and selection, validation of atmospheric turbulence prediction model, free space laser communication, and laser beam propagation in the atmosphere. The astronomical and adaptive optics communities have established several optical techniques for remote sensing Fried parameter, a parameter of pathintegrated structure constant of refractive index of atmosphere, but mote sensing ranged-resolved information of atmospheric turbulence. Laser guide stars technique is used commonly in adaptive optics community, and differential image motion method is a matured technique in astronomical community. So, a new technique is put forward for remote sensing atmospheric turbulence profiles. Combined with laser guide stars and differential image motion method, remote sensing of atmospheric turbulence profiles in real time is realized in experiments effectively.

The configure of experiment consist of a powerful pulsed laser, a beam-expanding lens and a negative lens for beam-focusing, a reflective mirror for beamprojecting, a optical device for receiving and imaging of returned signals, a ICCD camera for recording images of laser guide stars, and a computer for controlling and data processing. By expanding and focusing laser beam projected from a powerful pulsed lasers, laser guide stars are formed at several successive altitudes. A optical system with two receiving telescopes is developed based on differential image motion method. The laser guide stars are observed with the optical system developed, and images of laser guide stars with two spots at a receiving ICCD camera. The images with two spots at the same altitude are processed and centroids of two spots at every images are given. Differential image motion variance of distance of centroids could be derived from the images at the same altitude. So, the motion of the images of the laser guide stars from each altitude is characterized as the differential image motion variance.

Based on a inversion algorithm, atmospheric turbulence profiles could be retrieve from differential image motion variance of distance of centroids at various altitudes. Experiments of remote sensing have been performed, and the profiles of atmospheric turbulence are obtained. The structure constants of refractive index of atmosphere range from $10^{-14} \mathrm{~m}^{-2 / 3}$ at lower altitudes to $10^{-16} \mathrm{~m}^{-2 / 3}$ at higher altitudes. The results show it is a effective method that combined laser guide stars with differential image motion method and could sense atmospheric turbulence profiles remotely in real time and the profiles data remote sensed are reasonable.

\section{BACKGROUND AND PRINCIPLE of REMOTE SENSING}

\subsection{Background}

The atmospheric turbulence profiles had been measured in field by various methods, including radio-sonde with microthermal sensors, MASS(Multi-Aperture Scintillation Sensors) ${ }^{[1]}$, SCIDAR(SCIntillation Detection And Ranging) ${ }^{[2,3]}$, SODAR(SOund Detection And Ranging) ${ }^{[4]}$, and other techniques. The turbulence profiles measured by radio-sonde are not in real time and do not along vertical direction, the spatial resolution of turbulence 
profiles sensed by MASS are lower in vertical direction which are only seven layers, SCIDAR need binary stars within a certain angle, and SODAR only sense lower altitudes within boundary layers and altitude sensed is limited. In order to characterize the vertical structure of atmospheric turbulence, it is necessary to remote sense the whole turbulence profiles in real time.

It is a new technique to remote sense turbulence profiles by combined laser guide stars with differential image motion and its concept was put forward by M. S. Belen'kii et al ${ }^{[5]}$. In recent years, Georgia Tech Research Institute have developed brassboard turbulence LIDAR ${ }^{[6]}$ in 2007 and remote sensed turbulence profiles at horizontal path, in boundary layer measured and compared with microthermal sensors simultaneously, and along vertical paths in field. The layout of the brassboard turbulence LIDAR $^{[7]}$ is illustrated in Fig. 1, the example guide stars image ${ }^{[7]}$ is shown in Fig. 2, example of Differential Image Motion versus camera frame number is shown in Fig. 3, and the time-height diagram of the $\log$ of the strength of turbulence is shown in Fig. 4.

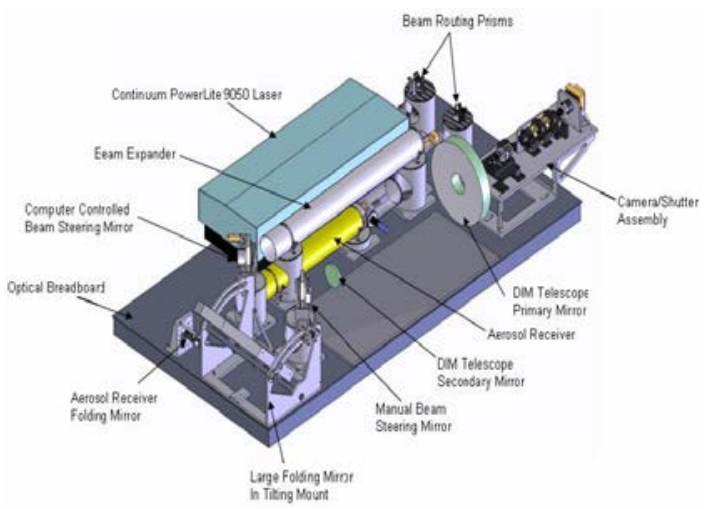

Fig. 1 Brassboard Turbulence LIDAR Layout

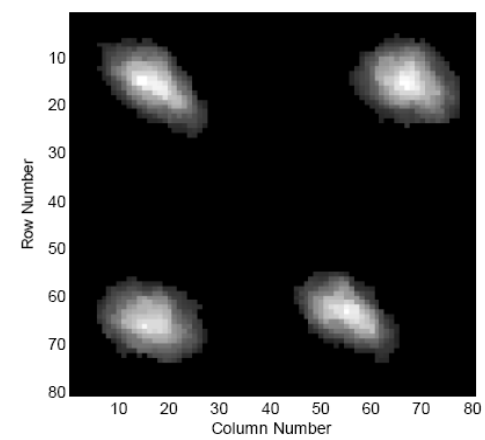

Fig. 2 Example Guide stars image

\subsection{Principle of remote sensing}

The Fried parameter could be sensed by differential image motion method(DIMM) ${ }^{[7]}$ utilizing a smaller telescope by place a mask with two holes in front of the telescope to remote sense a star. By this DIMM technique, the path-integrated parameter, Fried parameter, not a range-resolved profile, is sensed. If a star could be formed at any height and its brightness is enough, the range-resolved profiles of atmospheric turbulence could be sensed remotely in real time. So, a technique for remote sensing turbulence profiles of atmosphere is put forwards by combined differential image motion method and laser guide stars(LGS). Here differential image motion method is frequently-used and mature in astronomical community, and the method to form laser guide star is also frequently-used and mature in adaptive optics community. So, combining these two mature methods, a new technique "DIMM + LGS" is put forward for sensing turbulence profiles remotely in real time. The principle of remote sensing of atmospheric turbulence profiles is illustrated in Fig. $5^{[8]}$.

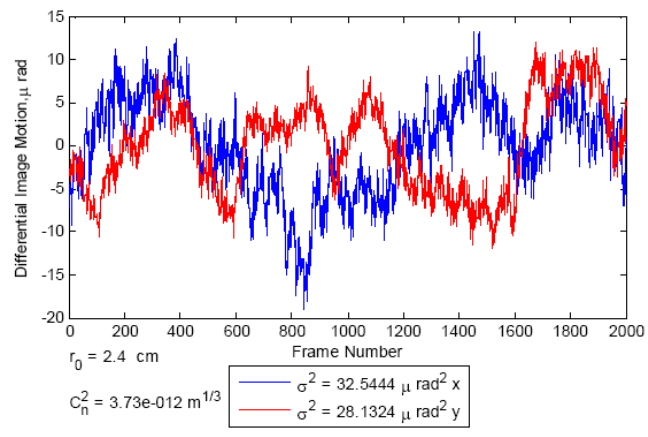

Fig. 3 example of Differential Image Motion versus camera frame number

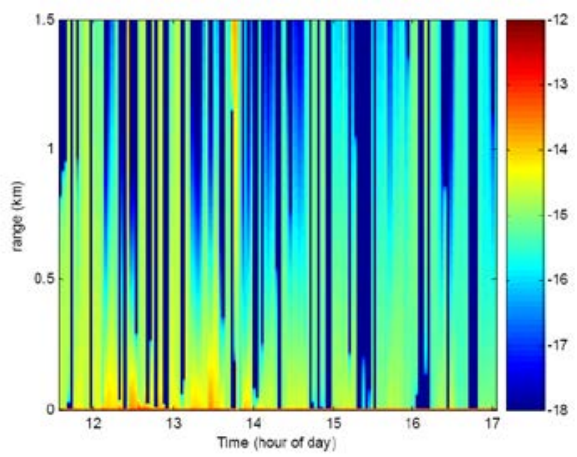

Fig. 4 Time-height diagram of the log of the strength of turbulence

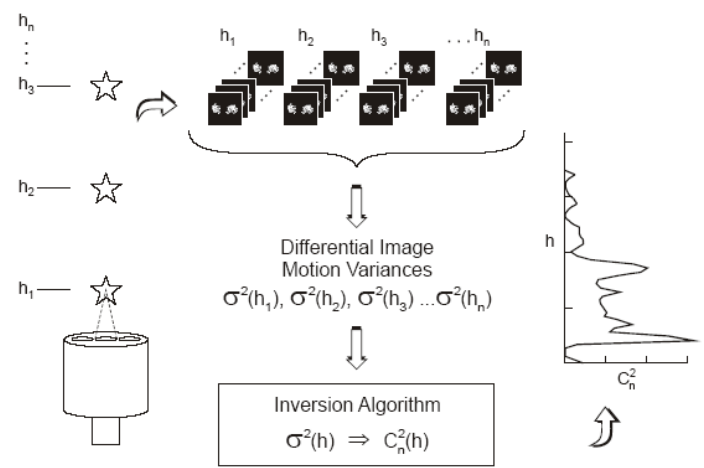

Fig. 5 The principle for remote sensing of atmospheric turbulence

As shown in Fig. 5, a powerful pulsed laser is focused and projected and laser guide stars are 
formed at various selected heights. The light from the scattering volume is received by a telescope with two spatially separated subapertures and the image of a laser guide star is separated to two images in the focal plane of ICCD camera by a wedge. Variance of centroids distance of images at various heights is obtained by the DIMM introduced above ${ }^{7}$, and a turbulence profile could be retrieved.

\section{REMOTE SENSING of TURBULENCE PROFILES}

\subsection{Experimental configuration}

Bases on "DIMM + LGS" technique, a experiment was carried out and the experimental configuration is illustrated in Fig. 6. The experiment system comprises four major parts: (1) the powerful pulsed laser which generates and transmites intense pulses of $532 \mathrm{~nm}$, duration about $6 \mathrm{~ns}$, and energy $200 \mathrm{~mJ}$ per pulse; (2) lens assembly for expanding, focusing, and projecting laser pulses, to form laser guide stars at selected heights; (3) a DIMM system for receiving signals of laser guide stars and forming images in focal plane of ICCD camera; (4) a computer for data processing to retrieve the profile of the atmospheric turbulence. The experimental configuration is offaxis, laser and lens assembly are placed on a platform in the top storey of a building, and laser pulses are transmitted from a circular hole in the ceiling of the building top and the aperture diameter 500The DIMM system placed above ceiling of the building top and could receive the returned signals of laser guide stars. After differential images and variance of centroids obtained, the computer processes the data, and retrieve profile of the atmospheric turbulence.

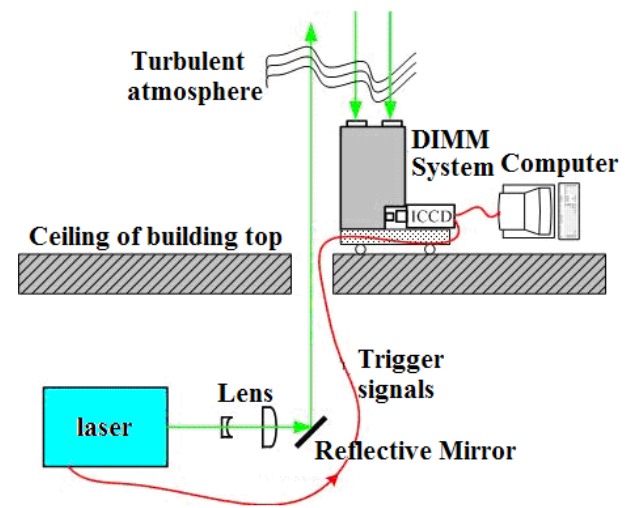

Fig. 6 Experimental configuration for remote sensing of turbulence profiles of atmosphere

\subsection{Experiment of remote sensing}

In the experiment for remote sensing turbulence profiles of atmosphere, firstly the pulsed laser is focused and transmitted, and then the laser guide stars are formed at pre-setting heights. After the returned signals of laser guide stars are received by the DIMM system, the differential motion images are formed in the focal plane of the ICCD camera, and the computer acquires the images, processes the data, and retrieves the turbulence profiles of atmosphere. At every heights, 2000 frames images should be acquired.

\subsection{Data processing and a typical result}

After images of laser guide stars are acquired, the locations of two centroids of spots in every images are calculated and distance between centroids are presented. Variance of differential image motion, $\sigma_{\text {DIM }}{ }^{2}$, is calculated utilizing $N($ here $N=2000)$ data at every heights. The Fried parameter could be calculated based on variance of differential image motion, and turbulence profile is retrieved based on variance of differential image motion at various heights. A typical data is presented in table 1 . The values of turbulence strength are descending along with increasing heights in negative exponential mode.

Table 1 Data of Remote Sensing and Turbulence Profile Retrieved

\begin{tabular}{cccc}
\hline Height/m & $\begin{array}{c}\text { Number } \\
\text { of Frames }\end{array}$ & $\sigma_{\text {DIM }}^{2} / \mathrm{rad}^{2}$ & $\mathrm{C}_{\mathrm{n}}^{2} / \mathrm{m}^{-2 / 3}$ \\
\hline 200 & 2000 & $6.53 \mathrm{e}-12$ & $2.75 \mathrm{e}-15$ \\
350 & 1999 & $3.27 \mathrm{e}-11$ & $1.47 \mathrm{e}-14$ \\
500 & 1966 & $1.31 \mathrm{e}-10$ & $5.69 \mathrm{e}-14$ \\
650 & 1982 & $1.36 \mathrm{e}-10$ & $1.27 \mathrm{e}-15$ \\
800 & 1983 & $2.02 \mathrm{e}-10$ & $3.68 \mathrm{e}-14$ \\
950 & 1988 & $2.12 \mathrm{e}-10$ & $5.90 \mathrm{e}-15$ \\
1100 & 1982 & $2.85 \mathrm{e}-10$ & $4.06 \mathrm{e}-14$ \\
1250 & 1976 & $3.00 \mathrm{e}-10$ & $8.87 \mathrm{e}-15$ \\
1400 & 1960 & $4.23 \mathrm{e}-10$ & $6.89 \mathrm{e}-14$ \\
1550 & 1934 & $5.40 \mathrm{e}-10$ & $6.56 \mathrm{e}-14$ \\
\hline
\end{tabular}

\section{CONCLUSIONS}

By analyzing the technique and remote sensing of turbulence profiles experimentally, it shows that turbulence profiles of atmosphere could be by "DIMM + LGS", and the results shows that the turbulence profile obtained is reasonable. By this technique, turbulence profiles could be sensed remotely in real time.

The validity of the data sensed remotely should be verification, and the data should be compared with the data measured with other method, such as microthermal sensors at certain heights.

In order to get $\mathrm{C}_{\mathrm{n}}{ }^{2}(h)$ at higher altitudes, the energy of per pulse of pulsed lasers should be powerful enough.

\section{REFERENCES}

[1] Kornilov, V., Tokovin, A., Vozyakova. O., "MASS: a monitor of the vertical turbulence distribution," . Proc. of SPIE 4839, 837-845(2003).

[2] Fuchs, A., Tallon, M., Vernin, J., "Focusing on a turbulence layer: principles of the generalized SCIDAR," Astronomical Society of the Pacific 110, 86-91(1998).

[3] Rodríguez-Hernández, M. A. C., Delgado, J. M., Fuensalida, J. J., et al., "The hybrid Shark- 
Hartmann/G-SCIDAR instrument," Proc. of SPIE 6747, 646712(2007).

[4] Travouillon, T., "SODAR calibration for turbulence profiles in TMT site testing," Proc. of SPIE 6267, 626720(2006).

[5] Belen'kii, M. S., Roberts, D. W., Stewart, J. M., Gimmestad, G. G., "Experimental validation of the differential image motion lidar concept," Optics Letters 25, 518-520(2000).

[6] Gimmestad, Gary G., Roberts, David A., Stewart, John M., et al., "LIDAR system for monitoring turbulence profiles," Proc. of SPIE 6551, 655110H(2006).

[7] Sarazin, M., Roddier, F., "The ESO differential image motion monitor," Astron. and Astrophys. 227, 294-300(1990).

[8] Gimmestad, Gary G., Roberts, David A., Stewart, John M., et al., "Testing of lidar system for turbulence profiles," Proc. of SPIE 6951, 6951109(2008). 